\title{
La sociologie politique et les expériences de modernisation des sociétés
}

\section{The Political Sociology and the Experiments in Modernization of Societies \\ La sociología política y las experiencias de modernización de las sociedades}

\section{S. N. EISENSTADT}

Volume 2, numéro 1, mai 1970

URI : https://id.erudit.org/iderudit/001227ar

DOI : https://doi.org/10.7202/001227ar

Aller au sommaire du numéro

Éditeur(s)

Les Presses de l'Université de Montréal

ISSN

0038-030X (imprimé)

1492-1375 (numérique)

Découvrir la revue

Citer cet article

EISENSTADT, S. N. (1970). La sociologie politique et les expériences de modernisation des sociétés. Sociologie et sociétés, 2(1), 25-42.

https://doi.org/10.7202/001227ar

\section{Résumé de l'article}

Après avoir signalé les ruptures et les continuités entre la théorie sociologique moderne et la pensée sociale et politique dont elle s'est progressivement détachée, l'auteur tente de situer la théorie politique par rapport à l'histoire de la sociologie politique occidentale et se propose de démontrer l'impact de la modernisation des pays non occidentaux sur la conceptualisation même des rapports entre la société civile et l'État, qui sont vus comme antinomiques dans la tradition européenne. Nous sommes conviés à relativiser cette conception et à reconnaître, à travers les explorations comparatives de la sociologie politique récente, l'émergence de questions nouvelles sur la nature des composantes de tout " centre societal " ou politique et, par conséquent, les variations possibles dans les affinités entre le pouvoir politique et le " centre-société ". Ces questions sont analysées ici en ce qu'elles contribuent à élargir les problèmes de la légitimation des systèmes politiques, de la participation à la sphère politique et du processus de sélection des élites politiques. 


\section{La sociologie politique et les expériences de modernisation des sociétés}

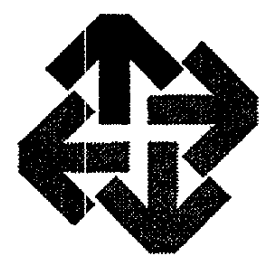

S. N. EISENSTADT

DANS Les PAGes qui suivent, je voudrais présenter une première analyse visant à démontrer partiellement comment l'extension du modernisme au-delà de l'ordre socio-politique et culturel occidental, et tout particulièrement en Asie, a nécessité une révision de nombreux concepts, et surtout des prémisses, de la sociologie politique moderne ${ }^{1}$.

Pour comprendre la démarche spécifique de la sociologie politique moderne, il serait bon d'analyser en premier lieu certaines des transformations et modifications qu'a subies toute la problématique de la nature de l'ordre social et politique et qui sont à l'origine du développement de la sociologie moderne à partir de l'examen de l'analyse philosophique.

L'importance grandissante accordée par la pensée sociale et politique à la distinction entre l'ordre civil et l'ordre politique constitue l'un des éléments de cette évolution. On perçoit de plus en plus nettement la société civile comme une

1. On trouvera un exposé plus complet de ces problèmes dans : S.N. Eisenstadt, * Sociological Thoughts, International Encyclopaedia of the Social Sciences, 1968; et dans l'introduction générale de son volume : The Scope and Problems of Political Sociology (à paraître à New York chez Basic Books Inc.) d'où a été tirée une grande partie de cet article. On trouvera une excellente analyse récente des principaux aspects du développement de la sociologie moderne dans : R. Aron, Main Currents in Sociological Thought, 2 vol., New York, Basic Books Inc., 1966-1967; Talcott Parsons, The Structure of Social Action, New York, Macmillan, 1937 et 1967. Cet ouvrage se situe parmi les plus importants exposés de la question. 
entité distincte et autonome qui n'est pas submergée par l'ordre politique ou «national », comme une force indépendante qui tendrait (selon les formulations théoriques postérieures) à ne considérer l'ordre politique lui-même que comme l'une des sphères institutionnelles de base de la société civile. La définition de la problématique de la volonté populaire donnée par Rousseau peut être considérée comme le tournant décisif vers cette façon de voir. Les noms de Lorenz von Stein, de Tocqueville ${ }^{2}$ et, d'une certaine manière, de Marx ${ }^{3}$, sont étroitement liés à la première phase de cette éviolution que développeront plus tard, de façon plus systématique et plus fouillée parallèlement à d'autres constatations, tous les grands noms de la pensée sociologique de la fin du $\mathrm{XIx}^{e}$ et du début du $\mathrm{Xx}^{\mathrm{e}}$ siècle : Spencer, Pareto, Durkheim, Max Weber et plus tard Karl Mannheim ${ }^{4}$.

Le deuxième point de départ important de l'investigation sociologique moderne se situe dans ce que l'on peut appeler la dissociation dialectique entre, premièrement, l'ordre transcendant et moral; deuxièmement, l'ordre socio-politique; et, troisièmement, l'ordre de la vie individuelle, c'est-à-dire des schémas dés comportements et des orientations individuels.

En premier lieu, l'investigation sociologique moderne découvre de plus en plus l'éventail de possibilités qu'offre le domaine de la transcendance et de la morale aux orientations et aux engagements individuels. Elle constate également que ces orientations et ces engagements ne sont qu'une des composantes du comportement individuel et, qu'au même titre que toutes les autres, elles se développent däns une lärge mesure à l'intérieur du' milieu "social; et qu'on se saurait donc attribuer les variations de ces composantes aux seules différences entre les individus.

En deuxième lieu vient la conscience grandissante de l'improbabilité qu'un ensemble d'institutions réalise entièrement le meilleur ordre moral possible ou représente pleinement un ớdre traniscendant; et, par voie de conséquence, l'improbábilité qu'on puisse évaluer les divers types de régimes sociaux ou politiques par rapport à ces types idéaux.

Parmi les précurseurs de ces orientations figurent, bien entendu, Hobbes et Rousseau et, jusqu'à un certain point, Locke ${ }^{5}$. Ce sont eux qui, en émettant l'hypothèse de l'existence possible d'un ordre social sans luii attribuer le caractère d'un phénomène naturel, ont posé le problème en des termes modernes même si les réponses concrètes qu'ils apportaient demeuraient fortement influencées par des

2. Lorenz von Stein, Staat und Gesellschaft, Zurich, Rascher, 1934; Alexis de Tocqueville, Democracy in America, New York, Knopf, 1954; Alexis de Tocqueville, l'Ancien Régime et la Révolution, traduit par N.W. Patterson, Oxford, Basil Blackwell, 1937.

3. Karl Marx, The Communist Manifesto, traduction anglaise, Chicago, C.H. Kerr, 1888; Karl Marx, Capital; New York, The Modern Library, 1936.

4. Herbert Spencer, Essays : Moral Political and Aesthetic, New York, Appleton, 1871; Vilfredo Pareto, The Mind and Society, Londres, J. Cape, 1935; Emile Durkheim, Professional Ethics and Civil Morales, Londres; Routledge and Kegan Paul, 1957; Max Weber, The Theory of. Social and Economic Organization, New York, Oxford University Press, 1947; Karl Mannheim, Ideology and Utopia, Londres, Routledge and Kegan Paul, 1936; Karl Mannheim, Man and Society in an Age of Reconstruction, Londres, Routledge and Kegan Paul, 1940.

5. Thomas Hobbes, The Leviathan, Oxford, Basil Blackwell, " Blackwell Political Texts *, 1946; Jean-Jacques Rousseau, The Social Contract, Londres, Sonnenschein, 1895; Jean-Jacques Rousseau, 'Political Writings, New York; Thomas, Nelson and Sons, 1953; John Locke, Two Treatises of Government, Cambridge, Cambridge University Press, 1960. 
modes de pensée anciens. Les apports ultérieurs des moralistes écossais ont joué ici un rôle encore plus déterminant.

En troisième lieu, on trouve à l'origine de la pensée sociologique moderne, la reconnaissance de plus en plus nette de l'existence d'une grande diversité de types: d'ordres sociaux et de leur variabilité interne, et la reconnaissance de la dimension temporelle (historique) comme une déterminante, sinon la seule, de cette diversité et variabilité.

La constatation de la diversité des modes sociaux, ou plutôt politiques, remonte au moins jusqu'à Aristote tout comme la recherche du rapport entre cette diversité et les divers types de comportements civiques et de positions morales des individus. À ces deux points de vue, la pensée sociologique moderne s'intègre largement dans la tradition aristotélicienne. Cependant, dans sa problématique, elle dépasse ce courant à cause de l'incorporation des deux points de vue précédents, Ainsi dépasse-t-on Aristote, premièrement en refusant d'assimiler l'ordre social à l'ordre politique et deuxièmement en soulignant la diversité des rapports entre l'engagement moral et l'orientation transcendantale, et les modes d'ordre social. À ce propos, comme le signale Shils, « la sociologie a partiellement scellé l'écart laissé par Aristote entre l'éthique et la politique ${ }^{7}$. La troisième considération, mais sans doute la plus importante, est que la pensée sociologique moderne dépasse Aristote en s'efforçant d'incorporer ou de tenir compte du développement temporel comme l'un des principaux facteurs de la variété et de la variabilité des divers types d'ordres sociaux.

En quatrième lieu, à l'origine de la théorie sociologique moderne, on trouve la reconnaissance de plus en plus répandue de l'influence des facteurs ambiants sur l'ordre social, en général, et la multitude des aspects de cet ordre, en particulier. Ici encore, les grands précurseurs sont Montesquieu, certains moralistes écossais, notamment Ferguson et Millar, plusieurs ethnologues et anthropologues, comme Tylor ${ }^{8}$ et, un peu plus tard, les diverses écoles évolutionnistes du XIX siècle quii seront suivies. par la grande poussée des études comparatives dans les sciences sociales au cours des années 40 de notre siècle.

De toutẻs ces manifestations de la perception des problèmes posés par l'ordre sociál, ont surgi plusieurs nouvelles tendances et orientations de la pensée idéologique, socio-philosophique ou plus spécifiquement sociologique: Cette dernière; tout en demeurant étroitement liée aux autres, a donné naissance, quoique laborieusement, à des problématiques propres que l'on peut formuler dans l'ensemble comme la recherche des conditions et des mécanismes de la continuité, de l'éclate-

6. Adam Smith, The Works of Adam Smith, 1963; Louis Schneider (édit.), The Scottish Moralists, Chicago, The University of Chicago Press, 1967.

7. Edward Shils, * The Calling of Sociology », dans Talcott Parsons, Edward Shils et al. (édit.), Theories of Society, New York, The Free Press of Glencoe, 1961, vol. 2, p. 1419.

8. Edward B. Tylor, Anthropology. An Introduction to the Study of Man and Civilization, Londres, Watts and $\mathrm{Co}, 1946$; et pour une histoire générale de l'anthropologie du point de vue de ses rapports avec ces problèmes, cf. R. Lowre, History of Ethnological Theory, New York, Holt, Rinehart and Winston, 1960. 
ment et du changement de l'ordre social, en général, ainsi que la variété des divers aspects de cet ordre, en particulier.

La problématique sociologique se caractérise par le refus de continuer à chercher les caractéristiques "naturelles » de l'ordre social ou la définition du meilleur modèle d'ordre social. Elle vise plutôt à analyser les conditions et les mécanismes de l'ordre social et de ses composantes, sa continuité et ses changements en général, et ceux de tel ou tel ordre social en particulier.

Dans le développement de la pensée sociologique, l'importance de la recherche des conditions et des mécanismes de l'ordre social, plutôt que des caractéristiques générales de la société comme un ordre « naturel » ou essentiellement moral, apparaît d'abord clairement dans le fait que les problèmes de désordre social, de désorganisation et de changement sont devenus le point central de la théorie sociologique, et ensuite, dans l'importance capitale qu'a prise le développement de certaines lois générales sur les conditions de l'ordre social dans diverses sociétés.

L'existence de conflits sociaux, l'ambiguïté des conflits internes et la démission des systèmes socio-politiques sont depuis longtemps reconnues (du moins depuis Platon et Aristote) comme un ensemble d'éléments à la base de toute société (ou régime politique) ou, selon Hobbes, comme un point de départ fondamental pour l'analyse de l'hypothèse d'un ordre social, en général. Si on se fonde sur ces phénomènes pour tenter de comprendre les mécanismes de l'ordre social, les conditions de fonctionnement et d'évolution de cet ordre en général et de ses diverses représentations en particulier, les préoccupations de la sociologie deviennent plus évidentes. Cela implique que le désordre social n'est pas antérieur à l'ordre social (et de ce fait différent) mais qu'il constitue un nouvel ensemble d'éléments qui, combinés différemment, sont à la base de la continuité de l'ordre social lui-même. Par conséquent, le chaos social peut devenir le point de départ d'une analyse sur le changement de cet ordre. Par ailleurs, cette théorie centre l'analyse sociologique sur la tendance vers la transformation non pas seulement de quelques événements externes ou isolés, mais des principaux aspects du phénomène de l'ordre social.

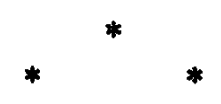

La problématique spécifique de la sociologie politique ou de l'investigation sociologique de l'ordre politique s'est développée à l'intérieur de ces préoccupations sociologiques modernes générales. Le développement de ces problématiques a subi l'influence d'un ensemble de conditions qui ont déterminé le progrès de la sociologie moderne. Celui-ci a été surtout influencé par le développement (à l'intérieur de la perception symbolique de l'ordre social par les groupes de l'intelligentsia européenne), d'une dichotomie grandissante dans la perception de l'\& État» et de la «Société 》 comme deux entités distinctes, relativement autonomes, mais pourtant étroitement liées. La dichotomie a particulièrement été signalée dans la pensée socio-politique allemande, et jusqu'à un certain point, dans la pensée française. De là, elle s'est répandue dans l'ensemble de la pensée et de l'investigation sociale depuis le début du XIX $X^{e}$ siècle. 
Cette conceptualisation de la dichotomie qui existe entre l'État et la Société a eu tendance à se développer suivant un mouvement pendulaire, oscillant entre des extrêmes dans l'histoire de la sociologie politique, souvent, mais pas toujours, en rapport avec des positions idéologiques différentes. Un extrême tendait à rabaisser la spécificité ou l'autonomie de la sphère des institutions et des processus politiques et cherchait à ne voir en eux que le reflet de forces sociales plus profondes qu'il s'agisse, comme dans le cas du marxisme, de structures économiques ou de classes, ou de l'«Esprit » général d'un pays, ou bien de diverses forces ambiantes ou technologiques. L'autre extrême, qui apparaît plus clairement dans ces tendances élitistes (Mosca ${ }^{9}$, Pareto et dans une certaine mesure Schumpeter), était porté à considérer les institutions politiques comme des sphères primordiales ou dominantes, et réduisait ainsi les autres sphères sociales à un rôle secondaire. Le clegré de prédominance idéologique de ces pôles était largement déterminé par les changements et les modes tant dans l'évolution de l'analyse sociologique que clans la recherche.

Les divers problèmes et tendances idéologiques, qui se sont polarisés autour de la dichotomie entre l'État et la Société, ont évolué dans de nombreuses directions au fil des décennies dans plusieurs pays. Comme nous l'avons vu, la perception de cette dichotomie était au tout début beaucoup plus forte et beaucoup mieux formulée en Allemagne et en Europe orientale que dans l'hémisphère occidental ou aux États-Unis. Pendant des périodes relativement longues, elle n'a pas été le point central de la pensée socio-politique, même dans les premiers pays. Mais elle a resurgi avec une vigueur nouvelle et sous des formes neuves et très brillantes avec la montée du totalitarisme, et spécialement de l'autoritarisme de droite (fascisme et nazisme). Au cours de cette période, les plus grands artisans de cette renaissance du problème ont sans doute été d'une part, Karl Mannheim, Edward Heiman 10 et le groupe des socialistes religieux et des idéologistes des diverses factions totalitaires, d'autre part, Carl Schmitt ${ }^{11}$ dans la droite et Lénine et Staline ${ }^{12}$ dans la gauche.

Avec le développement de la pensée sociologique et de la recherche, la perception de 1'État et de la Société comme entités opposées s'est progressivement muée en une interprétation plus nuancée dans laquelle la Société elle-même se divisait en plusieurs éléments constitutifs (l'économie, l'organisation sociale, la famille); tandis que la sphère politique, ou l'ordre lui-même, apparaîssait de plus en plus comme un de ces éléments. Et pourtant, la perception d'une dichotomie entre l'État et la Société a, en effet, légué à la sociologie politique contemporaine une très large part de sa problématique et de ses hypothèses d'analyse de base.

9. Gaetano Mosca, The Ruling Class, New York, McGraw-Hill Co., 1939.

10. Karl Mannheim, Man and Society in an Age of Reconstruction; Edward Heiman, Communism, Fascism or Democracy, New York, W.W. Norton and Co., 1938; Edward Heiman, Freedom and Order, Lessons from the War, New York, Scribner, 1947.

11. Carl Schmitt, «The Concept of The Political", Archiv für Sozialpolitik, vol. 58, septembre 1927.

12. Voir, par exemple, V.I. Lenin, The State and Revolution, New York, International Publishers, 1932; Joseph Stalin, Foundations of Leninism, New York, International Publishers, 1932. 
Cette situation n'a pas disparu, bien qu'elle se soit quelque peu atténuée, dès que les divers courants de recherche et d'analyse en sociologie politique eurent donné naissance à leurs propres traditions de problèmes et de recherche ${ }^{13}$, qu'il s'agisse des courants traitant des aspects systématiques de l'organisation des systèmes politiques, de la sélection et la composition des élites, du comportement politique et des conflits ou de l'analyse comparative des systèmes politiques.

Mais, assez paradoxalement, une fois que ces diverses méthodes de recherche de l'analyse de la sociologie politique eurent convergé vers des préoccupations d'analyse de base communes, leur dette envers la perception de la dichotomie entre l'État et la Société est devenue plus évidente.

Un examen plus attentif de certaines façons d'aborder l'hypothèse analytique de base, ainsi que la discussion qui les entoure, fait davantage ressortir cette dépendance, particulièrement en ce qui concerne l'analyse du caractère sociologique du pouvoir, des relations, des organisations et des institutions politiques, comme un phénomène distinct de l'ordre social ${ }^{14}$.

Dans la sociologie politique moderne et contemporaine, cette analyse gravite autour de trois problèmes. L'un est la définition des caractéristiques ou attributs formels ou «statiques » du système politique; le deuxième, la nature de ses rapports, de ses apports et de ses emprunts à d'autres domaines de l'ordre social; et le dernier, les conditions de stabilité, continuité et changement d'un système politique en général et de divers types spécifiques de systèmes politiques, en particulier ${ }^{15}$.

En dépit d'une controverse très animée sur la nature du «politique » et de ses rapports avec les autres éléments de la société, on peut aujourd'hui discerner une assez grande concordance d'opinions dans les façons d'aborder ces problèmes, si l'on s'en tient à un minimum de définitions de base de la nature systémique des institutions politiques dans n'importe quelle société ou une de ses parties. Mais, comme nous le verrons, ce consensus n'éteint pas la polémique sur les thèses fondamentales de l'analyse sociologique des systèmes politiques; d'une certaine manière, il ne fait que souligner dans quelle mesure la controverse demeure enracinée dans la conception dichotomique de l'Etat et la Société.

Dans la plupart de ces orientations, l'exercice du pouvoir politique et la nécessité d'un ordre politique, sont nés de deux besoins organiques ou conditions prérequises de tout système social en général, et d'une société totale, en particulier. Il s'agit d'une part du maintien de l'ordre et d'une réglementation des forces et,

13. Il ne m'appartient pas de nommer ici toutes les études pertinentes. On trouvera un compte rendu des écrits jusqu'en 1955 environ dans : R. Bendix et S.M. Lipset, "Political Sociology. A Trend and Bibliography ", Current Sociology, vol. 6, no 2, 1957, p. 79-169; S.M. Lipset, "Political Sociology ", dans R.K. Merton et al. (édit.), Sociology Today, New York, Basic Books Inc., 1959, p. 81-127; S.N. Eisenstadt, The Scope and Problems of Political Sociology, introduction générale.

14. A ce propos, cf. S.M. Lipset, "Political Sociology », dans R.K. Merton et al. (édit.), Sociology Today; G. Almond et R. Powell, Comparative Politics. A Developmental Approach, Boston, Little, Brown and Co., 1966. Soulignons ici l'importance des études de Parsons sur le pouvoir et les systèmes politiques, réunies pour la plupart dans son livre : Sociological Theory and Modern Society, New York, The Free Press, 1967 (cf. surtout la $3 \mathrm{e}$ partie). Comme nous le verrons, il s'agit des indications les plus importantes des efforts de la sociologie politique pour élargir ses horizons.

15. Cf. G. Almond et R. Powell, op. cit.; D. Easton, The Political System, New York, John Wiley, 1966. 
d'autre part, de l'implantation de certains objectifs communs, perçus comme représentatifs des aspirations de la collectivité. De ce fait, ces orientations supposent a priori l'existence d'un système politique dans toute société; ce qui revient à dire qu'il n'existe pas de société capable de réaliser ce qu'elle (ou ses élites) définit comme des idéaux collectifs et d'assurer l'ordre intérieur et extérieur, sans disposer d'un mode légitime d'interaction permettant la réalisation des idéaux et le maintien de l'ordre ${ }^{16}$. De la même façon, les problèmes majeurs de tout système politique apperaissent comme la transformation du pouvoir en une autorité qui dicte les limites de l'utilisation légitime de ce pouvoir et la relation entre l'autorité et la réalisation des desseins collectifs. C'est au sujet de ces problèmes centraux que quelques-unes des principales controverses, étroitement liées à la perception de la dichotomie entre l'État et la Société, se manifestent dans la sociologie politique moderne.

Dans la plupart des travaux sur la sociologie politique on peut discerner une certaine tendance à relever une contradiction fondamentale entre l'exercice du pouvoir et la représentation ou la réalisation des idéaux ou des valeurs d'une société. La manifestation de cette tendance apparaît clairement dans la grande ambivalence envers le pouvoir que l'on trouve dans beaucoup de discours sociologiques (et idéologiques) et dans certaines des polémiques qui entourent les tentatives pour surmonter cette ambivalence.

La plupart de ces controverses semblent se concentrer sur le principe d'un consensus de base pour affirmer la légitimité d'un système politique quelconque, un consensus qui est en grande partie conçu selon un accord des gouvernés avec les cibjectifs formulés et présentés par les gouvernants. Plusieurs des plus intenses polémiques de la sociologie politique cherchent à savoir si une telle légitimité, c'est-à-dire l'acceptation des objectifs d'une unité politique et de ses prétendus besoins par ses membres, n'est pas autre chose qu'un artifice employé par ceux qui parviennent à monopoliser le pouvoir dans une société donnée, une sorte d' « opium du peuple». Ici, on a souvent prétendu que les présumés «besoins» d'une société de maintenir un ordre politique quelconque, ne sont pas véritablement inhérents à la nature véritable des problèmes de cette société, mais qu'ils sont des 《fabrications » présentées comme des valeurs essentielles par ceux qui veulent accaparer le pouvoir. Ce sont ces derniers, a-t-on ajouté, qui imposent des objectifs à la société en s'efforçant de les présenter comme des besoins essentiels. Par conséquent, le consensus qui semblerait se dégager de l'apparente allégeance à ces objectifs ne serait autre chose qu'une «fiction » imposée par les gouvernants, sans rapport avec des convictions profondes et indépendantes des gouvernés ${ }^{17}$. Poul: appuyer cette hypothèse on a souvent émis l'opinion que les gouvernés ne sont pas en mesure d'utiliser le pouvoir à leurs propres fins, et que toute intervention de ce genre affaiblirait le pouvoir des gouvernants.

16. Adaptation de S.N. Eisenstadt, The Political Systems of Empires, New York, The Free Press of Glencoe, 1963, chap. 1 .

17. On trouvera quelques-uns des principaux jalons de cette controverse dans : C.W. Mills, The Power Elite, New York, Oxford University Press, 1959, et dans la critique de ce travail par l'arsons, publiée dans son livre : Structure and Process in Modern Societies, New York, The Free Press, 1960; B. Moore, Political Power and Social Theory, Cambridge (Mass.), Harvard University Press, 1958; R. Dahrendorf, "Out of Utopia », American Journal of Sociology, vol. $64, \mathrm{n}^{\mathbf{0}} 3,1958, \mathrm{p}$. 115-127. On trouvera un résumé utile de ces controverses dans : J. Rex, Key Problems of Sociological Theory, Londres, Routledge and Kegan Paul, 1961. 
D'un point de vue analytique, ce qui précède est étroitement lié à l'hypothèse de l'existence, dans toute société, d'une certaine somme limitée de pouvoir ainsi qu'aux supposées difficultés à expliquer les possibilités de changement social et politique, si on utilise ce concept du pouvoir dans l'analyse d'un système. En termes plus généraux, cela suppose une conception selon laquelle l'ordre social considéré serait comme étranger aux désirs et idéaux des individus, quelque chose qui leur serait fondamentalement imposé. Cette conception parallèle fait suite à celle plus idéologique qui perçoit la dichotomie dans le rapport entre l'État et la Société. Ce parallélisme se vérifie dans le fait que, dans l'attitude analytique et idéologique, la dimension politique de l'ordre social et culturel d'une part, et la dimension apolitique de cet ordre d'autre part, ont souvent été conçues comme des entités quelque peu séparées, distinctes et autonomes. S'il arrive qu'elles soient en corrélation, les rapports sont souvent jugés dichotomiques, antithétiques et de caractère fixe.

La portée de ces hypothèses devait être mise à l'épreuve, quoique sporadiquement et timidement, par la nouvelle flambée d'intérêt pour l'analyse politique et sociologique comparée (qui est liée à l'intérêt croissant pour les régions dites sous-développées, les sociétés en voie de développement ou les «nouvelles nations 》), et pour les études d'histoire comparée.

Au début, on a abordé l'étude de ces sociétés en se fondant surtout sur la dichotomie entre État et Société, comme par exemple dans la recherche des conditions sociales (non politiques) pour le développement de divers régimes politiques, ou dans l'importance d'un état à parti unique pour créer la cohésion socio-nationale. Mais le développement de ces études a démontré clairement que la plupart de ces hypothèses ne convenaient pas aux problèmes de ces sociétés.

Cette constatation s'explique de deux façons relativement simples et reliées entre elles. L'une veut que dans beaucoup de ces sociétés, en particulier celles issues de sociétés tribales, on ne puisse prendre pour acquis l'existence même de l'État ou de la Société selon la notion européenne traditionnelle de deux éléments forts et autonomes. Dans un grand nombre de ces sociétés (notamment celles d'Afrique) il n'existait pas de structure politique pourvue d'un centre distinct. La plupart de ces sociétés montraient l'absence relative d'un centre caractéristique des sociétés primitives, tandis que la majorité des centres qui s'étaient créés avec elles étaient d'origine étrangère. De la même façon, dans ces cas on ne pouvait considérer comme acquise l'existence d'états correspondant à des communautés nationales et à des groupes politiques relativement homogènes.

En apparence, cette situation aurait pu amener ces états aux racines de la pensée politique européenne moderne, aux idées politiques de Hobbes, Rousseau ou Burke ${ }^{18}$, qui mettaient l'accent sur le problème du consensus et du développement d'une «volonté commune». Mais en réalité, la confrontation avec les problèmes de l'établissement d'un ordre social et politique moderne fut ici d'une

18. Ali A. Mazrui, "Edmund Burke and Reflections on the Revolution in the Congo ", Comparative Studies in Society and History, vol. 5, 1962-1963, p. 121-133. 
tout autre nature. Tous ces penseurs européens classiques considéraient comme acquise l'existence d'un État et d'une Société. Même lorsqu'ils s'effrayaient de l'anarchie ou de la guerre civile, ils envisageaient ces dangers dans le cadre d'une structure politique traditionnellement puissante et centralisée. Cependant, dans un grand nombre de nouveaux états, l'existence d'un pouvoir central viable et d'une communauté pantribale était très précaire; et les conditions pouvant aboutir à leur développement exigeaient un examen beaucoup plus approfondi. Le réexamen des conceptions de base de l'ordre politique était aussi rendu nécessaire par la rencontre avec un grand nombre de sociétés asiatiques dotées de fortes traditions impériales. Bien qu'il ne puisse y avoir de doute ici quant à la présence d'un dispositif spécifique d'État, les relations mêmes entre l'État et l'ordre social étaient envisagées de manières différentes de celles définies dans la tradition occidentale.

Cette tradition politique ne révélait pas le même genre de dichotomie entre État st Société que sa contrepartie européenne ${ }^{19}$. Elle tendait davantage à souligner la conformité de rapport entre l'ordre cosmique d'une part, et l'ordre socio-politique, d'autre part. Contrairement à la tradition occidentale, la relation entre la politique et le social n'était pas envisagée en termes d'antinomie entre deux unités ou deux puissances. On l'énonçait plutôt en termes d'une fusion de diverses fonctions à l'intérieur d'un même groupe ou organisme, centré sur un objectif commun dans l'ordre cosmique.

Ces développements et rencontres ont eu plusieurs répercussions sur notre perception de la nature de l'ordre politique et social et contribuèrent certainement à la révision et à la refonte de beaucoup des concepts ou, du moins, des hypothèses de base de la sociologie politique. En toute vérité, une telle révision ne fait que débuter, mais il convient de signaler quelques-unes des orientations possibles.

Ilci l'étape cruciale initiale est, bien entendu, la reconnaissance du fait que l'ordre socio-politique européen moderne n'a été qu'un des nombreux ordres socio-politiques modernes, ou du moins post-traditionnels, qu'il est permis d'envisager ${ }^{20}$. La forme la plus caractéristique de cet ordre socio-politique a été celle de l'État-nation tel qu'il est apparu en Europe (et plus tard aux États-Unis) pendant la pésiode postérieure à la Contre-Réforme, au cours du XVIII et du XIX siècle.

ILes caractéristiques dominantes de ce genre d'ordre socio-politique ont été a) un niveau élevé de conformité entre l'identité culturelle et l'identité politique de la population du territoire, $b$ ) un niveau élevé d'engagement symbolique et affectif envers le centre et le lieu de l'intégration territoriale et $c$ ) une volonté

19. Voir, à ce propos, Reinhard Bendix, Nation-Building and Citizenship, New York, John Wiley, 1964. Voir aussi des études récentes sur certains aspects de l'œuvre de Marx par rapport à l'Asie, par exemple : George Litchtheim, Marx and the Asiastic Mode of Production ", St. Anthony's Papers, ${ }^{\circ}$ 14, 1963; Daniel Thorner, "Marx on India and the Asiatic Mode of Production , Contributions to Indian Sociology, $\mathrm{n}^{\circ}$ 9, décembre 1966, p. 3-66 (contient une bibliographie complète de cette controverse); F. Tokei, "Sur le mode de production asiatique , Studia historica Academia scientiarum hungartical, Budapest, Akadémiai Kiado, 1966.

210. Pour plus de détails, voir l'introduction à la section sur les sociétés modernes, dans S.N. Eisenstadt, The Scope and Problems of Political Sociology. 
marquée de définir politiquement des objectifs collectifs pour tous les membres de la communauté nationale.

À plus d'un égard plusieurs des caractéristiques de l'état-nation européen proviennent des divers secteurs de traditions socio-politiques prémodernes, de traditions impériales ${ }^{21}$ et de celles des cités-États des sociétés féodales ${ }^{22}$. Elles ont assimilé les fortes orientations activistes de la cité-État, la vision large d'un ordre politique activement relié à l'ordre cosmique ou culturel des traditions impériales, et les éléments pluralistes de la tradition féodale. En Europe, il y a eu plusieurs orientations qui ont abouti à un ordre socio-culturel et politique qui se fondait tant sur un niveau relativement élevé d'engagement de divers groupes à l'ordre culturel et politique et à ses centres, que sur un fort degré d'autonomie dans l'accès à cet ordre et à ses centres respectifs ${ }^{23}$.

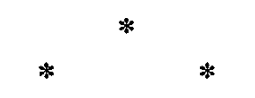

Mais l'expansion du modernisme, c'est-à-dire la rupture de la légitimité traditionnelle de l'ordre socio-politique et culturel, et la recherche, au-delà de l'Europe, d'un ordre socio-politique fondé sur une légitimité extra-traditionnelle, l'amena en contact avec des sociétés civilisées dont la tradition politique était faite de modes et d'orientations fondamentales différentes. Par exemple, dans les sociétés impériales asiatiques (en particulier l'Empire chinois) les éléments pluralistes étaient beaucoup plus faibles que dans les sociétés féodales ou les cités-États. Chez beaucoup d'autres peuples (du sud-est asiatique, d'Afrique et, dans une certaine mesure, d'Amérique latine) les forces du modernisme ont empiété sur des systèmes patrimoniaux où le niveau d'engagement à l'ordre socio-politique, aussi bien que l'étroit rapport entre l'ordre politique et l'ordre cosmique, était beaucoup plus faible ${ }^{24}$.

Un grand nombre de ces sociétés se caractérisent par la faiblesse des centres qui, tout en s'acquittant de leurs fonctions techniques (comme les tâches de politique externe ou administratives du centre politique, ou des tâches rituelles et théologiques d'un centre religieux), n'entretiennent que très peu de rapports autonomes avec d'autres centres auxquels ils n'ont guère accès et sur lesquels ils n'exercent qu'un contrôle négligeable. Des centres de ce genre ne peuvent acquérir de la puissance des autres centres ou ordres de vie sociale et culturelle. Ils ne peuvent pas davantage s'acquitter adéquatement de certaines fonctions possibles de direction et de légitimation et, de ce fait, ne s'attirent qu'un minimum d'engagement au-delà des limites de leur propre rayonnement, et quelquefois même de l'intérieur. Leurs relations avec d'autres centres, groupes ou couches plus vastes ont surtout un

21. Cf. S.N. Eisenstadt, The Political Systems of Empires.

22. A propos du féodalisme, cf. J. Prawer et S.N. Eisenstadt, « Feudalism », International Encyclopaedia of the Social Sciences. A propos des cités-Etats et des ordres féodaux, cf. S.N. Eisenstadt, The Scope and Problems of Political Sociology.

23. A propos de ces caractères spécifiques du système européen, cf. S.N. Eisenstadt, The Political System of Empires; S.N. Eisenstadt, * Prestige, Participation and Strata-Formation », dans J.A. Jackson (édit.), Sociological Studies, Cambridge, Cambridge University Press, 1967, en particulier p. 95-100.

24. Pour plus de détails sur les systèmes patrimoniaux, cf. $\mathrm{M}$. Weber, The Theory of Social and Political Organization, New York, Bedminster Press, 1968, vol. 3, p. 1006-1070. 
caractère adaptatif (comme dans le cas de nombreux conquérants nomades par rapport aux organisations religieuses des peuples conquis), et ces centres peuvent tant symboliquement qu'effectivement, être entièrement submergés, comme certains centres religieux du sud-est asiatique qui ont presque complètement été absorbés par les centres politiques.

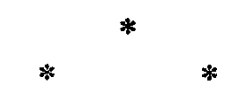

Les remarques précédentes n'épuisent pas les nombreuses variantes des sphères socio-politiques et culturelles traditionnelles et post-traditionnelles en dehors de l'Europe. Mais il n'appartient pas à cet article de tenter une explication exhaustive de ces divers types. Nous voudrions plutôt analyser l'effet de la constatation de cette diversité de régimes socio-politiques sur la formulation de quelques-uns des problèmes fondamentaux de la sociologie politique. Cette étude exige au préalable une définition plus poussée des divers éléments ou composantes de tout centre sociétal ou politique qui, comme nous le verrons plus tard à la lumière de l'expérience européenne, ont eu tendance à se développer parallèlement. Parmi ces composantes, la plus importante a toujours été l'institutionnalisation, aussi bien en termes symboliques qu'organisationnels, de la recherche d'un moyen d'ordonner l'experrience sociale et culturelle et d'une participation à de tels ordres charismatiques.

D'un caractère distinct, mais étroitement liée à cette recherche, on trouve la deuxième composante d'un centre en formation : la cristallisation de l'identité collective sociétale et culturelle autour des attributs communs ou autour d'une participation à des événements symboliques collectifs. C'est cet élément qui fournit la base des facteurs "précontractuels 》 d'un ordre social.

Une troisième composante de la formation d'un centre (qui peut en fait se rattacher étroitement tant au développement des éléments d'une identité commune qu'à l'institutionnalisation d'un ordre plus vaste mais qui demeure analytiquement distinct) est la cristallisation et la formulation d'objectifs communs, d'objectifs « organisationnels » qui sont représentés comme ceux de la collectivité ou du régime politique.

Une quatrième composante de la formation de centres (très souvent mentionnée dans la littérature sociologique et non moins souvent considérée en étroite relation avec le niveau de différenciation sociale et de spécialisation) consiste en la réglementation des rapports au sein de la société et entre les groupes, c'est-à-dire la façon de traiter les pseudo-problèmes d'intégration causés par la complexité croissante de la division sociale du travail.

Une cinquième composante de la formation de centres se présente sous forme de réglementation des rapports de forces internes et externes. Assez paradoxalement, l'importance de la force - ou de la puissance pure dans ses aspects coercitifs dans les relations internes et particulièrement les relations systématiques externes d'un système, n'a pas fait l'objet d'une analyse rationnelle ou assez nuancée de la part de la sociologie politique. Elle a été tantôt négligée, tantôt exagérée. Il est vrai que la réglementation des forces a toujours été un élément de base de tout système politique; mais dans la plupart des cas cet élément a été considéré comme la composante essentielle ou prédominante de tout système politique, ou comme 
un problème que chaque système est appelé à résoudre, ou tout au plus comme un moyen d'atteindre les objectifs essentiels d'un régime politique. Même si ces composantes sont considérées comme des facteurs de base de l'activité politique ou comme lui étant étroitement liées, on n'a pas encore étudié à fond leur interaction systématique en termes d'analyse générale ou comparée.

Le fait d'estimer, ainsi que nous venons de le faire, chacun de ces éléments comme essentiel dans la formation d'un centre et capable d'engendrer un type spécial de relation sur le plan plus large de l'ordre social et culturel et de circonscrire des problèmes spécifiques d'organisation, facilite notre compréhension de la dynamique des systèmes sociaux et politiques. Cette analyse révèle d'abord que chacun des éléments ou composante d'un centre se rapporte, d'une part, à divers problèmes institutionnels et, d'autre part, aux orientations relatives à la conduite « charismatique » qu'un centre doit observer, à des degrés et selon des modes variés; elle indique aussi que, bien que ces composantes puissent exister dans chaque centre, leur influence relative dans la constitution du centre peut varier.

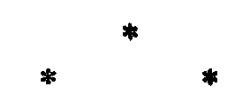

La reconnaissance des diverses implications de ces constatations dépasse nécessairement l'expérience et la tradition de l'ordre socio-politique moderne européen où la plupart des éléments constitutifs d'un centre tendent à se fondre dans un cadre organisationnel et institutionnel et une perspective commune. Cette tradition suppose l'existence de centres forts, et assume également, à l'intérieur des centres politiques, toutes les composantes de formation qui existent dans un ordre donné et qui obéissent à des modes fixes ou des rapports antinomiques. Elle tend en particulier à mettre en valeur l'importance de la réglementation des rapports des groupes et des forces, et, de la relation étroite aux symboles charismatiques de l'ordre social et culturel.

Le dépassement de cette tradition soulève plusieurs problèmes nouveaux, en premier lieu celui de la place spéciale qu'occupe le domaine politique par rapport au centre-société. Il semble à première vue que l'organisation politique centrale constitue le centre «naturel» de toute société, quelle que soit sa forme ou sa composition. Mais cela ne signifie pas nécessairement que le centre ou l'institution politique d'une société soit le seul point de convergence ou d'attrait. Pourtant, il ne fait aucun doute qu'il existe une relation spéciale, quoique non exclusive, entre le domaine politique et ce point central. Le domaine politique, celui de l'autorité, constitue le domaine privilégié où sont réunis les éléments d'ordre de la force charismatique; c'est un point important de référence dans n'importe quel centre sociétal. Cette place spéciale du régime d'institution politique comme pôle de centralisation, et la grande affinité entre la centralité et la sphère politique, a sa racine dans la nature de l'autorité. D'une certaine façon, comme nous l'avons vu, l'essence du politique est la transformation du pouvoir en autorité, laquelle transformation a ses origines dans l'affinité qui existe entre la politique, les symboles et les qualités charismatiques du centre. Par conséquent, l'institution politique entretient 
un double rapport avec la recherche de l'ordre. D'abord, elle est le moyen d'organisation qui canalise les exigences et les besoins de cet ordre ainsi que ceux du lieu institutionnel de réglementation du pouvoir; ensuite elle joue un rôle déterminant dans l'établissement du centre ou des centres de l'ordre social et culturel.

Cependant, l'analyse qui précède révèle également qu'à l'intérieur de plusieurs systèmes politiques l'affinité des institutions politiques à la «centralité » charismatique peut être très mince et, qu'à sa place, d'autres composantes du centre peuvent prédominer. La combinaison de ces composantes peut aussi être plutôt faible et ténue. Cette analyse peut jeter une lueur nouvelle sur le caractère des tensions inhérentes aux centres ou ordres politique, social et culturel. Elle démontre que la tension n'est pas seulement attribuable à l'antinomie, souvent soulignée par la pensée occidentale, entre l'organisation et l'exercice du pouvoir et la participation au maintien d'un vaste ordre socio-culturel. Bien que cette antinomie puisse largement contribuer à créer les tensions, celles-ci proviennent également du fait que les qualités charismatiques de l'ordre social - et la volonté d'y participer ne sont pas concentrées dans une seule sphère institutionnelle mais qu'elles se dispersent, quoique différemment, dans toutes les sphères. Ce phénomène peut expliquer, dans une certaine mesure, à la fois l'existence d'un pluralisme d'autorités dans une société, les propensions naturelles des détenteurs du pouvoir politique à tenter de monopoliser et de réglementer le centre, et leur désir intime d'y parvenir. Cette tension peut s'expliquer aussi par le fait que chaque centre, qu'il soit politique, culturel ou social, se compose (à différents degrés) de plusieurs éléments qui entretiennent des rapports différents avec la dimension charismatique de l'ordre social et qu'il peut avoir à résoudre des problèmes institutionnels ou organisationnels différents.

Cette analyse des rapports entre la politique et les diverses formes de centralité et d'autorité peut nous aider à corriger certaines interrogations fondamentales de la soriologie politique, en particulier celles qui se rapportent à la légitimation des systèmes politiques, à la recherche de participation à la sphère politique, et au processus de sélection des élites politiques.

Par un curieux paradoxe, l'étude précédente révèle que la nature et l'étendue de cette légitimation dans le fonctionnement des systèmes politiques sont à la fois plus larges et plus étroites que ne l'a souvent supposé la sociologie politique. Elle élargit l'horizon de la légitimation et lui fait embrasser un domaine de composantes beaucoup plus étendu. Elle la rend moins dépendante de l'efficacité d'un système politique dans la réglementation des rapports entre les groupes ou de ses affinités avec certaines conceptions de l'ordre et de sociétés plus vastes. De plus, elle étend l'éventail des critères de légitimation à toutes les composantes de la formation de centres mentionnées ci-dessus.

Mais, simultanément, elle réduit l'importance de la légitimation dans la continuité des systèmes politiques, du point de vue des éléments de formation d'un centre. Ou, mieux encore, au lieu de considérer comme un postulat la relation 
entre la légitimation du point de vue de ces composantes avec l'affinité et la continuité d'un système politique, elle fait de cette relation une variable.

Comme le problème de la légitimation des systèmes politiques est étroitement associé à l'opinion voulant que la stabilité ou la continuité d'un système politique dépende du consensus sur sa légitimité, l'analyse qui précède indique aussi quelques mesures nécessaires pour reformuler la place qu'occupe le consensus dans le fonctionnement des systèmes politiques. Elle montre en premier lieu la nécessité de préciser la portée et la base du consensus, c'est-à-dire de déterminer dans quelle mesure un système politique quelconque semble «acceptable » à ceux qui y participent, tant du point de vue de son efficacité d'organisation, de son orientation charismatique plus vaste et de son effet de polarisation, que de celui de n'importe quelle composante de la formation d'un centre. En deuxième lieu, elle pose le problème de l'acceptation des différences ou de l'évaluation d'un régime politique par les divers participants du système. Enfin, elle soupèse l'influence des divers niveaux de consensus sur la stabilité, la continuité et les possibilités de changement dans un système politique.

La nécessité d'une nouvelle formulation vise également plusieurs autres problèmes primordiaux de la sociologie politique, principalement ceux qui se rapportent à la nature de la lutte et de la participation politiques ainsi que de la sélection des élites. L'analyse qui précède indique que la présentation de la recherche habituelle de participation à la vie politique (et par voie de conséquence à la lutte politique) comme un désir de participer à la distribution du pouvoir et aux décisions d'autorité quant à la répartition des biens et des ressources dans la société n'est que partiellement fondée.

Lorsqu'on constate l'étroite relation entre l'autorité et le centralisme et leurs diverses composantes, la recherche de participation dans le domaine politique doit être envisagée non seulement comme une recherche pour le plaisir dans le sens organisationnel étroit du terme, mais également comme une participation aux sphères vitales de la société et dans l'ordre plus vaste et plus significatif que lui confèrent son importance et son caractère charismatique plutôt que dans quelque dérivé organisationnel ou institutionnel des autres composantes du centre. Ainsi la sélection d'élites politiques doit-elle être considérée non seulement d'après l'efficacité pure ou la lutte pour le pouvoir, mais aussi selon d'autres composantes, qu'il s'agisse de l'aptitude des élites à réorganiser l'ordre symbolique et réel inhérent aux orientations plus larges de ce genre et à l'ordre institutionnel qui les englobe, ou de toute autre composante des centres.

Certains éléments de la formation des centres sont mis en valeur ou combinés de manière différente selon les élites. L'examen des conditions dans lesquelles différents types d'élites peuvent naitre constitue l'un des problèmes les plus difficiles qui confrontent la sociologie politique.

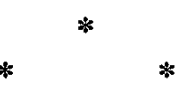

La redéfinition des rapports entre la charismatique, la formation des centres et les institutions et un processus politique, peut aussi nous aider à faire un examen de quelques-uns des principaux attributs du symbolisme, de la réflexion et de la 
philosophie politique, et peut-être également de leurs rapports avec la sociologie politique. Dans toutes les cultures, les symboles et la pensée politique se concentrent sur les rapports entre l'ordre politique et les autres types d'ordres charismatiques (cosmique, moral et social), et plus particulièrement sur les ordres perçus, selon la tradition d'une société ou d'une culture donnée, comme les indices les plus représentatifs de la culture de base et de l'identité collective, et les paramètres les plus précis de l'existence humaine. Les pôles essentiels de cette relation sont généralement envisagés du point de vue de la pertinence symbolique mutuelle et organisationnelle de ces ordres, de leur légitimité, autonomie et responsabilité.

Les autres ordres (apolitiques) sont-ils très pertinents par rapport à l'ordre politique? Et, selon le degré de leur pertinence, comment sont-ils conçus et comment perçoit-on et organise-t-on ces rapports ? L'ordre politique incarne-t-il l'ordre cosmique, ou n'en est-il qu'un appendice secondaire ? Où se trouve le lieu symbolique central de l'ordre général plus vaste ? Est-ce dans les domaines politique ou cosmique, moral ou religieux ? Quelle est l'importance de la dimension politique pour l'existence humaine à l'échelle cosmique? Jusqu'où peut aller l'autonomie symbolique et organisationnelle, virtuelle ou réelle, des divers centres dans lesquels ces ordres variés se trouvent? Et, consécutivement, quel est le lieu de légitimation et de' responsabilité de l'ordre politique ? Quels rapports existent entre ces facteurs et les problèmes spécifiques de structuration de l'organisation politique et de ses fonctions, et avec les différentes composantes de la formation d'un centre ? Enfin, quelles sont les relations de ces facteurs avec les exigences d'organisation des autres domaines sociaux?

Un deuxième point de convergence du symbolisme et de la pensée politique se trouve dans la façon de percevoir les rapports entre le centre (ou les centres) et la périphérie, ainsi que le mode d'accès et de participation à la périphérie des groupes ou des couches plus vastes dans les divers centres en général, et dans le centre politique en particulier. Cette manière de voir est, par définition, très fortement reliée à la conception fondamentale et à l'image du centre politique et de ses rapports avec les autres centres, c'est-à-dire de l'ordre politique dans ses rapports avec les autres ordres. Il est intéressant de remarquer ici la valeur relative attribuée, dans une société donnée, aux diverses composantes des centres et la mesure dans laquelle la participation à leurs activités, définie comme distincte de la participation à d'autres aspects des composantes du centre, est reconnue comme importante.

Le troisième point de concentration important de tout symbolisme et rhétorique politique est ce qu'on pourrait appeler le schéma de comportement concernant les dirigeants et les dirigés; celui du bon dirigeant et du bon sujet, ou citoyen.

Ici, deux problèmes fondamentaux surgissent. L'un a trait à la définition de ces rapports réciproques du point de vue du contenu de base de l'ordre prédominant, de la nature fondamentale des symboles charismatiques au centre d'une tradition, et de l'aptitude ou de la volonté des dirigeants comme des gouvernés de respecter, dans le domaine politique, les préceptes de cet ordre. Le deuxième problème important est le rapport entre le schéma idéal de comportement des gouvernants et des gouvernés, de leurs obligations mutuelles et de leurs relations réciproques 
découlant des principes de base du « charismatique » avec les exigences de l'organisation politique et l'efficacité administrative. Dans presque toutes les philosophies et rhétoriques politiques, les conditions requises des composantes structurales d'un centre sont entièrement reconnues et explorées; qu'il s'agisse des composantes du jeu politique, c'est-à-dire la nécessité pour le gouvernant de maintenir un certain équilibre entre les diverses factions de la société dans son ensemble ou dans son entourage privé, ou de l'efficacité relative de l'administration. Mais la nature des rapports aux niveaux les plus profonds de l'ordre et du comportement politique approprié est conçu différemment selon les tradition culturelles.

Les différences se rapportent à l'importance que l'on attribue aux composantes d'un centre (l'administration technique, le politique, la réglementation des rapports entre les groupes, etc.) dans l'existence individuelle, et à la mesure dans laquelle une des composantes domine davantage les autres. Dans la plupart des régimes qui ont précédé l'ère moderne l'autonomie relative de l'ordre politique était perçue en fonction du degré de développement d'un certain particularisme qui, assez paradoxalement, se combinait, comme aux Indes, avec le rituel et l'idéologique, mais sans que le «sacré » domine l'organisation et sans que l'importance de la dimension politique de la vie diminue.

Mais, quelles que soient ces différences, dans la plupart des traditions on limite à trois domaines les points de concentration principaux autour desquels les relations entre le «technique » et l' « organisationnel », et les aspects plus idéologiques ou symboliques du domaine politique tendent à se cristalliser. Le premier tend à percevoir la nature des règles et des normes de la lutte politique. Le deuxième évalue la nature et l'importance du degré selon lequel le système politique doit satisfaire les groupes plus larges de la société, qu'il s'agisse du niveau matériel (ou de sécurité) ou du niveau «charismatique» d'une portée plus large. Enfin, le troisième cherche dans quelle mesure la participation à la vie politique, dans l'une des composantes du centre, représente un but important à l'intérieur de l'ensemble des buts et des désirs de l'individu.

Dans une paraphrase quelque peu différente, pour reprendre la terminologie de Rousseau, l'un des points centraux de la rhétorique politique est la façon de concevoir les rapports entre la volonté commune et la «volonté de tous »; dans quelle mesure existe-t-il, à l'intérieur de la volonté individuelle de personnes différentes, une vision commune du bien de tous ? et dans quelle mesure le bien commun est-il interprété en termes politiques et de participation à l'ordre politique ?

De plus, le point central de tous ces problèmes a été en général la recherche d'un «bon» régime politique et $\mathrm{du}$ «bon» gouvernant, citoyen ou sujet. Mais cette «bonté » n'a pas toujours été conçue de la même façon par les cultures et les civilisations humaines. Les réponses à ces problèmes et la façon de les formuler ont grandement varié selon les traditions. À l'intérieur de ce vaste panorama de traditions diverses de réflexion sur des questions politiques, la méthode occidentale n'en est qu'une parmi beaucoup d'autres.

Dans cette tradition, l'intérêt philosophique pour l'institution politique a été justifié dans une large mesure par la recherche du bien moral, défini surtout en termes séculiers, privés ou civiques. De ce fait, le bien politique est comparé à la bonté morale des individus, et le comportement politique à un bon comportement 
moral. Cette façon de formuler le problème des rapports entre les domaines politique, culturel et moral, et surtout d'apporter les solutions spécifiques à ces problèmes, est particulière à la civilisation occidentale. Dans beaucoup d'autres civilisations, les critères de la morale individuelle ont été très secondaires dans l'évolution de l'orclre social et politique et des rapports entre les deux. La morale est tout au plus perçue comme la manifestation d'une conception plus profonde de la bonté, ou comme une bonté d'un autre ordre.

Un examen plus approfondi des diverses perceptions symboliques de l'ordre politique et des conditions dans lesquelles elles se développent peut nous aider à élucider les problèmes de la sociologie politique qui découleraient de la révision des critères qui a été tentée ci-dessus.

\section{RÉSUMÉ}

Après avoir signalé les ruptures et les continuités entre la théorie sociologique moderne et la pensée sociale et politique dont elle s'est progressivement détachée, l'auteur tente de situer la théorie politique par rapport à l'histoire de la sociologie politique occidentale et se propose de démontrer l'impact de la modernisation des pays non occidentaux sur la conceptualisation même des rapports entre la société civile et l'Etat, qui sont vus comme antinomiques dans la tradition européenne. Nous sommes conviés à relativiser cette conception et à reconnaître, à travers les explorations comparatives de la sociologie politique récente, l'émergence de questions nouvelles sur la nature des composantes de tout * centre sociétal s ou politique et, par conséquent, les variations possibles dans les affinités entre le pouvoir politique et le * centre-société ». Ces questions sont analysées ici en ce qu'elles contribuent à élargir les problèmes de la légitimation des systèmes politiques, de la participation à la sphère politique et du processus de sélection des élites politiques.

\section{ABSTRACT}

[The Political Sociology and the Experiments in Modernization of Societies] After having pointed out the continuities and discontinuities between modern sociological theory and the political and social thought from which it grew, the author attempts to place political theory in perspective with the history of social movements in Western politics itself. Further, he proposes to demonstrate the impact modernization in non-Western countries has had on the concept of the relationship between State and society which in the European tradition is seen as antinomic. We are asked to consider this idea in context and to recognize, in the light of recent comparative studies in political sociology, that new questions have arisen about the way nature of the components of social and political centers tendencies and consequently about the possible variations in the affinity between State and society. These questions are analyzed here insofar as they add to the problems of political system legitimation, of political participation, and of the selection of political elites.

\section{RESUMEN}

[La sociología politica y las experiencias de modernización de las sociedades] Despues de señalar las rupturas y las continuidades que presenta la teoría sociológica moderna con respecto al pensamiento social y político del cual se ha separado progresivamente, el autor trata de situar la teoría política con respecto a la historia de la sociología política occidental y propone una lectura del impacto de la extensión del modernismo en los países no occidentales sobre la conceptualización misma de las relaciones entre la sociedad civil y el Estado, relaciones interpretadas como antinómicas en la tradición europea. Se sugiere relativizar esta concepción y reconocer, a traves los estudios comparativos de la sociología política reciente, la emergencia de nuevas cuestiones sobre la naturaleza de los componentes de todo a centro societal o político $y$, por consiguiente, las variaciones posibles en las afinidades entre el poder político y el centro sociedad . Tales cuestiones son analizadas en todo lo que contribuyen a extender los problemas de la legitimación de los sistemas políticos, de la participación en la esfera política y del proceso de selección de la flor política. 